\title{
Dynamics of non-archimedean Polish groups
}

\author{
Alexander S. Kechris*
}

\begin{abstract}
.
A topological group $G$ is Polish if its topology admits a compatible separable complete metric. Such a group is non-archimedean if it has a basis at the identity that consists of open subgroups. This class of Polish groups includes the profinite groups and $\left(\mathbb{Q}_{p},+\right)$ but our main interest here will be on non-locally compact groups.

In recent years there has been considerable activity in the study of the dynamics of Polish non-archimedean groups and this has led to interesting interactions between logic, finite combinatorics, group theory, topological dynamics, ergodic theory and representation theory. In this paper I will give a survey of some of the main directions in this area of research.
\end{abstract}

2010 Mathematics Subject Classification. Primary 03C15, 22F50, 54H20, 37A15.

Keywords. Non-archimedean groups, Fraïssé theory, Ramsey theory, ample genericity, automatic continuity, unique ergodicity, spatial realizations, unitary representations.

\section{Non-archimedean groups and automorphism groups}

Below $S_{\infty}$ is the infinite symmetric group, i.e., the group of all permutations of $\mathbb{N}$. This is a Polish group with the pointwise convergence topology (when $\mathbb{N}$ is endowed with the discrete topology). The following is a basic fact about non-archimedean Polish groups (see, e.g., [5], 1.5).

Theorem 1.1. Let $G$ be a Polish group. Then the following are equivalent:

(i) $G$ is non-archimedean,

(ii) $G$ is isomorphic (as a topological group) to a closed subgroup of $S_{\infty}$.

Remark 1.2. Another characterization of non-archimedean Polish groups (that also explains the terminology) is that they are exactly those that admit a compatible left-invariant ultrametric (see again [5], 1.5).

A more useful for our purposes way to view the preceding characterization is through some basic concepts of model theory.

Let $L$ be a countable first-order language, i.e., a countable family $L=\left\{R_{i}\right\}_{i \in I} \sqcup$ $\left\{f_{j}\right\}_{j \in J} \sqcup\left\{c_{k}\right\}_{k \in K}$ of relation, function and constant symbols, where each relation symbol $R_{i}$ has an associated arity $n_{i} \geq 1$ and each function symbol $f_{j}$ has an

\footnotetext{
*Work on this paper was partially supported by NSF Grant DMS-0968710. I would like to thank J. Melleray, L. Nguyen Van Thé, C. Rosendal, M. Sokić, S. Solecki, S. Thomas and T. Tsankov for many helpful comments on an earlier draft of this paper.
} 
associated arity $m_{j} \geq 1$. A structure for $L$ has the form

$$
\boldsymbol{A}=\left\langle A,\left\{R_{i}^{\boldsymbol{A}}\right\}_{i \in I},\left\{f_{j}^{\boldsymbol{A}}\right\}_{j \in J},\left\{c_{k}^{\boldsymbol{A}}\right\}_{k \in K}\right\rangle,
$$

where $R_{i}^{\boldsymbol{A}} \subseteq A^{n_{i}}, f_{j}^{\boldsymbol{A}}: A^{m_{j}} \rightarrow A, c_{k}^{\boldsymbol{A}} \in A$. We call $A$ the universe of the structure $\boldsymbol{A}$ and we say that $\boldsymbol{A}$ is countable (resp., finite) if $A$ is countable (resp. finite). We put $|\boldsymbol{A}|=|A|=$ cardinality of $A$.

For such a structure $\boldsymbol{A}$, we let $\operatorname{Aut}(\boldsymbol{A})$ be its automorphism group. When $\boldsymbol{A}$ is countable, this is again a Polish group with the pointwise convergence topology ( $A$ being discrete) and clearly it is isomorphic to a closed subgroup of $S_{\infty}$. Conversely if $G \leq S_{\infty}$ is a closed subgroup, we associate with it its so-called canonical structure, $\boldsymbol{A}_{G}=\left\langle\mathbb{N},\left\{R_{i}^{\boldsymbol{A}_{G}}\right\}_{i \in I}\right\rangle$, which is defined as follows:

Consider $G$ acting on each $\mathbb{N}^{n}(n \geq 1)$ coordinatewise and let $I_{n}^{G}$ be the set of orbits. Let $I=\bigsqcup_{n \geq 1} I_{n}^{G}$ and for $i \in \bar{I}_{n}^{G}$, let $R_{G, i}$ be a relation symbol of arity $n$. Let $L=\left\{R_{G, i}\right\}_{i \in I}$. Then $R_{i}^{\boldsymbol{A}_{G}}=i \subseteq \mathbb{N}^{n}$ for $i \in I_{n}^{G}$.

It is easy to see that $G=\operatorname{Aut}\left(\boldsymbol{A}_{G}\right)$, so we have the following characterization.

Theorem 1.3. Let $G$ be a Polish group. Then the following are equivalent:

(i) $G$ is non-archimedean,

(ii) $G$ is isomorphic to the automorphism group of a countable structure.

\section{Fraïssé theory}

The canonical structure $\boldsymbol{A}_{G}$ associated to a closed subgroup $G \leq S_{\infty}$ has an important symmetry property, called ultrahomogeneity.

Given a structure $\boldsymbol{A}$ in a language $L$ a substructure $\boldsymbol{B}$ of $\boldsymbol{A}$, in symbols $\boldsymbol{B} \subseteq \boldsymbol{A}$, is any structure of the form $\boldsymbol{B}=\left\langle B,\left\{R_{i}^{\boldsymbol{B}}\right\}_{i \in I},\left\{f_{j}^{\boldsymbol{B}}\right\}_{j \in J},\left\{c_{k}^{\boldsymbol{B}}\right\}_{k \in K}\right\rangle$, where $B \subseteq A, B$ contains all $c_{k}^{\boldsymbol{A}}, k \in K$, and is closed under all $f_{j}^{\boldsymbol{A}}, j \in J$, and $R_{i}^{\boldsymbol{B}}=R_{i}^{\boldsymbol{A}} \cap B^{n_{i}}, f_{j}^{\boldsymbol{B}}=f_{j}^{\boldsymbol{A}} \mid B^{m_{j}}, c_{k}^{\boldsymbol{B}}=c_{k}^{\boldsymbol{A}}$. A partial isomorphism of $\boldsymbol{A}$ is an isomorphism $\pi: \boldsymbol{B} \rightarrow \boldsymbol{C}$, where $\boldsymbol{B}, \boldsymbol{C} \subseteq \boldsymbol{A}$. The structure $\boldsymbol{A}$ is called ultrahomogeneous if any isomorphism between finite substructures of $\boldsymbol{A}$ can be extended to an automorphism of $\boldsymbol{A}$. Typical ultrahomogeneous structures include the rational order, the random graph, the countable atomless Boolean algebra, the countably infinite-dimensional vector space over a finite field, etc.

It is now easy to check that $\boldsymbol{A}_{G}$ is ultrahomogeneous, so we have the following sharper version of 1.3 .

Theorem 2.1. Let $G$ be a Polish group. Then the following are equivalent:

(i) $G$ is non-archimedean,

(ii) $G$ is isomorphic to the automorphism group of a countable ultrahomogeneous structure.

Fraïssé theory provides an analysis of countable ultrahomogeneous structures in terms of their finite substructures, which is the key to many interesting connections between the study of the dynamics of automorphism groups and logic, finite combinatorics and other areas. 
A countable structure $\boldsymbol{K}$ is a Fraïssé structure if it is infinite, locally finite (i.e., finitely generated substructures are finite) and ultrahomogeneous. As usual we say that a substructure $\boldsymbol{A}$ of a structure $\boldsymbol{B}$ is finitely generated if there is a finite set $X \subseteq A$ such that $\boldsymbol{A}$ is the smallest substructure of $\boldsymbol{B}$ with $X \subseteq A$. If there are only finitely many constant symbols and no function symbols in the language of $\boldsymbol{K}$, then local finiteness is automatically satisfied, so the canonical structures $\boldsymbol{A}_{G}$ introduced earlier are Fraïssé structures. Thus, up to isomorphism, the Polish non-archimedean groups are exactly the automorphism groups of Fraïssé structures. Moreover the examples of ultrahomogeneous structures mentioned earlier are Fraïssé structures.

Given a structure $\boldsymbol{A}$, let $\operatorname{Age}(\boldsymbol{A})$ be the class of all finite structures $\boldsymbol{B}$ that can be embedded into $\boldsymbol{A}$, i.e., Age $(\boldsymbol{A})=\{\boldsymbol{B}: \boldsymbol{B}$ finite, $\boldsymbol{B} \leq \boldsymbol{A}\}$, where $\boldsymbol{B} \leq \boldsymbol{A}$, i.e., $\boldsymbol{B}$ can be embedded into $\boldsymbol{A}$, means that $\boldsymbol{B}$ is isomorphic to a substructure of $\boldsymbol{A}$. Such an isomorphism is called an embedding of $\boldsymbol{B}$ into $\boldsymbol{A}$.

When $\boldsymbol{K}$ is a Fraïssé structure, the class $\mathcal{K}=\operatorname{Age}(\boldsymbol{K})$ of finite structures has the following properties:

(i) Hereditary Property (HP): If $\boldsymbol{A} \in \mathcal{K}$ and $\boldsymbol{B} \leq \boldsymbol{A}$, then $\boldsymbol{B} \in \mathcal{K}$.

(ii) Joint Embedding Property (JEP): If $\boldsymbol{A}, \boldsymbol{B} \in \mathcal{K}$, there is $\boldsymbol{C} \in \mathcal{K}$ with $\boldsymbol{A} \leq$ $\boldsymbol{C}, \boldsymbol{B} \leq \boldsymbol{C}$.

(iii) Amalgamation Property (AP): If $\boldsymbol{A}, \boldsymbol{B}, \boldsymbol{C} \in \mathcal{K}$ and $f: \boldsymbol{A} \rightarrow \boldsymbol{B}, g: \boldsymbol{A} \rightarrow \boldsymbol{C}$ are embeddings, then there is $\boldsymbol{D} \in \mathcal{K}$ and embeddings $r: \boldsymbol{B} \rightarrow \boldsymbol{D}, s: \boldsymbol{C} \rightarrow \boldsymbol{D}$ with $r \circ f=s \circ g$.

(iv) $\mathcal{K}$ is countable up to isomorphism, i.e., there are only countably many isomorphism types in $\mathcal{K}$.

(v) $\mathcal{K}$ is unbounded, i.e., contains structures of arbitrarily large finite cardinality.

Any class $\mathcal{K}$ of finite structures (in the same language $L$ ) satisfying (i)-(v) is called a Frä̈ssé class. Thus when $\boldsymbol{K}$ is a Fraïssé structure, $\operatorname{Age}(\boldsymbol{K})$ is a Fraïssé class. The key result of the Fraïssé theory is the converse. Fraïssé showed that one can associate to each Fraïssé class $\mathcal{K}$ a canonical Fraïssé structure $\boldsymbol{K}$ called its Fraïssé limit, $\boldsymbol{K}=\operatorname{Flim}(\mathcal{K})$, which is the unique Fraïssé structure whose age is equal to $\mathcal{K}$. Therefore one has a canonical one-to-one correspondence $\mathcal{K} \mapsto \operatorname{Flim}(\mathcal{K})$ between Fraïssé classes and Fraïssé structures, whose inverse is $\boldsymbol{K} \mapsto \operatorname{Age}(\boldsymbol{K})$. This establishes an important duality theory between Fraïssé classes and Fraïssé structures. Here are some examples of this correspondence:

(i) The Fraïssé limit of the class of finite graphs is the random graph.

(ii) The Fraïssé limit of the class of finite linear orderings is the rational order.

(iii) The Fraïssé limit of the class of finite-dimensional vector spaces over a finite field is the countably infinite-dimensional vector space over that field.

(iv) The Fraïssé limit of the class of finite Boolean algebras is the countable atomless Boolean algebra.

(v) The Fraïssé limit of the class of finite metric spaces with rational distances is the rational Urysohn space $\mathbb{U}_{0}$. (The completion of this space is the Urysohn space $\mathbb{U}$ - see, e.g., [30].) 


\section{The main directions}

In the dynamics of non-archimedean groups, we will concentrate in this paper in three main directions:

A) Topological dynamics, which will be subdivided into two main topics:

I) Universal minimal flows, extreme amenability and structural Ramsey theory.

II) Generic symmetries and automatic continuity phenomena.

B) Ergodic theory, which will be subdivided into two main topics:

I) Unique ergodicity phenomena.

II) Spatial realizations.

C) Unitary representations.

\section{Topological dynamics, I: Universal minimal flows, extreme amenability and structural Ramsey theory}

A) We will start by reviewing some standard concepts of topological dynamics.

Let $G$ be a (Hausdorff) topological group. A $G$-flow is a continuous action of $G$ on a (Hausdorff, nonempty) compact space $X$. A subflow of $X$ is a (nonempty) compact invariant set with the restriction of the action. A flow is minimal if it has no proper subflows or equivalently every orbit is dense. It is easy to see (by an application of Zorn's Lemma) that every $G$-flow contains a minimal subflow.

A homomorphism between two $G$-flows $X, Y$ is a continuous $G$-map $\pi$ : $X \rightarrow Y$ (i.e., $\pi(g \cdot x)=g \cdot \pi(x), \forall g \in G, x \in X)$. Note that if $Y$ is minimal $\pi$ must be a surjection. Finally an isomorphism between $X, Y$ is a bijective homomorphism. 1).

The following is a classical result in topological dynamics (see, e.g., [30], Section

Theorem 4.1. For any topological group $G$, there is a minimal $G$-flow, $M(G)$, with the following property: For any minimal $G$-flow $X$, there is a homomorphism $\pi: M(G) \rightarrow X$. Moreover, $M(G)$ is the unique, up to isomorphism, minimal $G$-flow with this property.

The flow $M(G)$ is called the universal minimal flow of $G$. If $G$ is compact, $M(G)=G$, with the left translation action. However, if $G$ is locally compact but not compact, $M(G)$ is extremely complicated, e.g., it is not metrizable (see [30], Appendix 2). For example, when $G$ is discrete, for instance, $G=\mathbb{Z}, M(G)$ is a "big" space of ultrafilters on $G$. However, it is a remarkable phenomenon that for groups $G$ that are not locally compact, $M(G)$ can even trivialize, i.e., be a singleton. 
This leads to two general problems in topological dynamics:

(i) When is $M(G)$ trivial?

(ii) Even if $M(G)$ is not trivial, when can one explicitly determine $M(G)$ and show that it is metrizable?

These problems have been extensively studied in the last thirty years or so. Let us first give the following definition.

Definition 4.2. A topological group $G$ is called extremely amenable if its universal minimal flow $M(G)$ is trivial.

This is equivalent to saying that $G$ has an extremely strong fixed point property: Every $G$-flow has a fixed point. For that reason, sometimes extremely amenable groups are also said to have the fixed point on compacta property. Note also that this condition is a significant strengthening of the concept of amenability, which asserts that every $G$-flow admits an invariant probability Borel measure.

Mitchell [41] raised the question of the existence of (non-trivial) extremely amenable groups and Granirer-Lau [21] and Veech [63] showed that no (non-trivial) locally compact group can be extremely amenable. The first examples of extremely amenable groups were produced by Herer-Christensen [23], who showed that there are Polish abelian groups with no non-trivial unitary representations, sometimes called exotic groups. Such groups are extremely amenable. The first natural example of an extremely amenable group was produced by Gromov-Milman [22], who showed that the unitary group of the infinite-dimensional separable Hilbert space has this property. This proof used methods of asymptotic geometric analysis related to the phenomenon of concentration of measure that we will not discuss in this paper. By such methods, other important examples were discovered later:

(i) Furstenberg-Weiss (unpublished), Glasner [14]: The group $L(X, \mu, \mathbb{T})$ of all measurable maps from a standard measure space $(X, \mu)$ into $\mathbb{T}$,

(ii) Pestov [50]: The isometry group Iso(U) of the Urysohn space $\mathbb{U}$,

(iii) Giordano-Pestov [13]: The automorphism group, $\operatorname{Aut}(X, \mu)$, of a standard measure space $(X, \mu)$.

Pestov [49] also produced another example, namely Aut $(\langle\mathbb{Q},<\rangle)$. However his proof did not use concentration of measure techniques but rather finite combinatorics, more specifically the classical Ramsey theorem. From this it also follows that the group $H_{+}([0,1])$ of increasing homeomorphisms of $[0,1]$ is also extremely amenable.

We will next discuss the study of extreme amenability and the calculation of universal minimal flows for non-archimedean Polish groups represented as automorphism groups of Fraïssé structures $\boldsymbol{K}$. This was undertaken in the paper Kechris-Pestov-Todorcevic [30]. The main outcome of this study is the development of a duality theory which shows that there is an "equivalence" between the structure of the universal minimal flow of $\operatorname{Aut}(\boldsymbol{K})$ and the Ramsey theory of the class of finite structures $\mathcal{K}=\operatorname{Age}(\boldsymbol{K})$.

B) We will review first some basic concepts in an area of finite combinatorics known as structural Ramsey theory. 
For natural numbers $N \geq m \geq k \geq 1, r \geq 1$, the notation $N \rightarrow(m)_{r}^{k}$ means that for every coloring (map) $c:[N]^{k} \rightarrow\{1, \ldots, r\}$, where $[N]^{k}=\{A \subseteq$ $\{1, \ldots N\}:|A|=k\}$, there is a subset $X \subseteq\{1, \ldots, N\}$ with $|X|=m$ which is monochromatic, i.e., $c$ is constant on $[X]^{k}=\{A \subseteq X:|A|=k\}$. The classical Ramsey theorem is the assertion that given $m \geq k \geq 1, r \geq 1$ there is large enough $N \geq m$ such that $N \rightarrow(m)_{r}^{k}$. Notice that this can be also equivalently stated in terms of finite linear orderings. Given finite linear orderings $\boldsymbol{K} \leq \boldsymbol{M}$ and $r \geq 1$, there is a linear ordering $\boldsymbol{N} \geq \boldsymbol{M}$ such that if we color all suborderings of $\boldsymbol{N}$ isomorphic to $\boldsymbol{K}$ with $r$ colors, there is a monochromatic copy of $\boldsymbol{M}$ in $\boldsymbol{N}$, i.e., an isomorphic to $\boldsymbol{M}$ subordering of $\boldsymbol{N}$ so that all its suborderings isomorphic to $\boldsymbol{K}$ have the same color.

Structural Ramsey theory is a vast extension of the classical Ramsey Theorem to classes of finite structures. It was developed primarily in the 1970's by Graham, Leeb, Rothschild, Nešetřil-Rödl, Prömel, Voigt, Abramson-Harrington and others (see, e.g., Nešetřil [43] or [30] for some references).

Let $\mathcal{K}$ be a class of finite structures in a language $L$. For $\boldsymbol{A} \leq \boldsymbol{B}$ in $\mathcal{K}$ let

$$
\left(\begin{array}{l}
B \\
A
\end{array}\right)=\left\{A^{\prime} \subseteq B: A^{\prime} \cong \boldsymbol{A}\right\}
$$

be the set of isomorphic copies of $\boldsymbol{A}$ contained in $\boldsymbol{B}$. For $\boldsymbol{A} \leq \boldsymbol{B} \leq \boldsymbol{C}$ in $\mathcal{K}$ and $r \geq 1$, let

$$
\boldsymbol{C} \rightarrow(\boldsymbol{B})_{r}^{\boldsymbol{A}}
$$

mean that for any coloring $c:\left(\begin{array}{l}\boldsymbol{C} \\ \boldsymbol{A}\end{array}\right) \rightarrow\{1, \ldots, r\}$, there is $\boldsymbol{B}^{\prime} \in\left(\begin{array}{l}\boldsymbol{C} \\ \boldsymbol{B}\end{array}\right)$ such that $c$ is constant on $\left(\begin{array}{l}\boldsymbol{B}^{\prime} \\ \boldsymbol{A}\end{array}\right)$. We say that $\mathcal{K}$ has the Ramsey Property (RP) if for any $\boldsymbol{A} \leq \boldsymbol{B}$ in $\mathcal{K}$ and $r \geq 1$, there is $\boldsymbol{C} \geq \boldsymbol{B}$ in $\mathcal{K}$ with $\boldsymbol{C} \rightarrow(\boldsymbol{B})_{r}^{\boldsymbol{A}}$.

Among the many examples of classes with the RP that are now known (see the above references), we can mention the following:

(i) finite linear orderings (Ramsey),

(ii) finite Boolean algebras (Graham-Rothschild [20]),

(iii) finite-dimensional vector spaces over a given finite field (Graham-LeebRothschild [19],

(iv) finite ordered graphs (Nešetřil-Rödl [45]

(v) finite ordered metric spaces (Nešetřil [44]).

However the class of finite graphs and the class of finite metric spaces do not have the RP.

Now we can summarize in general terms the duality theory alluded to earlier. Let $\mathcal{K}$ be a Fraïssé class and $\boldsymbol{K}=\operatorname{Flim}(\mathcal{K})$ its Fraïssé limit. Then we have a "canonical" correspondence between: (i) the structure of the universal minimal flow of $\operatorname{Aut}(\boldsymbol{K})$ and (ii) the Ramsey theory of $\mathcal{K}$.

We will next discuss specific instances of this duality and some of their applications.

C) We will first consider the problem of characterizing the extremely amenable non-archimedean Polish groups. They are of the form $G=\operatorname{Aut}(\boldsymbol{K})$ for a Fraïssé 
structure $\boldsymbol{K}$. Let $\mathrm{LO}(K)$ be the space of all linear orderings on the universe $K$ of $\boldsymbol{K}$. This is a compact subspace of $2^{K^{2}}$ with the product topology and $G$ acts continuously on it as follows: if $<\in \mathrm{LO}(K)$ and $g \in G$, then $g \cdot\langle\in \mathrm{LO}(K)$ is defined by $x(g \cdot<) y \Leftrightarrow g^{-1} \cdot x<g^{-1} \cdot y$. Thus, if $G$ is extremely amenable, there is a fixed point $<$ for this action, i.e., a linear ordering on $K$ invariant under $\operatorname{Aut}(\boldsymbol{K})$. Thus $\operatorname{Aut}(\boldsymbol{K})=\operatorname{Aut}(\langle\boldsymbol{K},<\rangle)$, so we can assume to start with (by replacing $\boldsymbol{K}$ by $\langle\boldsymbol{K},<\rangle)$ that $\boldsymbol{K}$ is an order Fraïssé structure, i.e., $\boldsymbol{K}$ has the form $\boldsymbol{K}=\langle K,<, \ldots\rangle$ where $<$ is a linear ordering on $\boldsymbol{K}$. In this case $\mathcal{K}=\operatorname{Age}(\boldsymbol{K})$ is an order Fraïssé class, i.e., each $\boldsymbol{A} \in \mathcal{K}$ has the form $\boldsymbol{A}=\langle A,<\ldots\rangle$, where $<$ is a linear ordering on $A$. Here are some examples of order Fraïssé classes:

(i) finite linear orderings,

(ii) finite ordered graphs,

(iii) finite ordered metric spaces with rational distances,

(iv) (Thomas [58]) lexicographically ordered finite-dimensional vector spaces over a finite field (i.e., ordered vector spaces in which the order is induced lexicographically by an ordering of some basis),

(v) lexicographically ordered finite Boolean algebras (i.e., ordered Boolean algebras in which the ordering is induced lexicographically by an ordering of its atoms),

(vi) finite posets with linear extensions.

Thus we see that the extremely amenable non-archimedean groups are to be found among the $\operatorname{Aut}(\boldsymbol{K}), \boldsymbol{K}$ an order Fraïssé structure. But which ones? The answer is given by the following:

Theorem 4.3 ([30]). Let $\mathcal{K}$ be an order Fraïssé class and $\boldsymbol{K}=\mathrm{Flim}(\mathcal{K})$ its Frä̈ssé limit. Then the following are equivalent:

(i) $\operatorname{Aut}(\boldsymbol{K})$ is extremely amenable,

(ii) $\mathcal{K}$ has the Ramsey Property.

Another formulation of this result which does not explicitly refer to ordered structures is the following, pointed out by Nguyen Van Thé [47].

Let $\mathcal{K}$ be a Fraïssé class and $\boldsymbol{K}=\operatorname{Flim}(\mathcal{K})$. Then the following are equivalent:

(i) $\operatorname{Aut}(\boldsymbol{K})$ is extremely amenable,

(ii) $\mathcal{K}$ consists of rigid structures and has the Ramsey Property.

We will next mention some applications of this result.

I) Using the extensive results of the structural Ramsey theory and 4.3, one can now produce many new examples of interesting extremely amenable groups. These include the automorphism groups of the structures: $\langle\mathbb{Q},<\rangle$ (the result of Pestov mentioned earlier); $\langle\boldsymbol{R},<\rangle$, where $\boldsymbol{R}$ is the random graph and $<$ is a linear ordering so that $\langle\boldsymbol{R},<\rangle$ is the Fraïssé limit of the class of ordered finite graphs, i.e., the random ordered graph; $\left\langle\boldsymbol{V}_{\infty, F},<\right\rangle$, the countably infinite-dimensional vector space over a finite field $F$ with the ordering induced by the Fraïssé limit of the lexicographically ordered finite-dimensional vector spaces; $\left\langle\boldsymbol{B}_{\infty},<\right\rangle$, the countable atomless Boolean algebra with the appropriate linear ordering as before; $\left\langle\boldsymbol{U}_{0},<\right\rangle$, the rational Urysohn space with the linear ordering induced by the Fraïssé limit 
of the linearly ordered finite metric spaces with rational distances, etc. References for these and many other examples can be found, for example, in [30], [46].

II) Although 4.3 is about non-archimedean groups, it can be applied to prove extreme amenability for other important Polish groups via dense homomorphisms. This is based on the following simple observation: Let $G, H$ be Polish groups and let $\pi: G \rightarrow H$ be a continuous homomorphism with $\pi(G)$ dense in $H$. If $G$ is extremely amenable, so is $H$. There are now situations under which automorphism groups $\operatorname{Aut}(\boldsymbol{K})$ of Fraïssé structures $\boldsymbol{K}$ can be mapped homomorphically and densely in the above sense to other Polish groups $H$, which are far from non-archimedean. Then one can reduce the proof of extreme amenability of $H$ to that of $\operatorname{Aut}(\boldsymbol{K})$. This has been used, for example, in [30] to give a new proof of the extreme amenability of Iso $(\mathbb{U})$ via finite combinatorics, as opposed to the original proof of Pestov that used analytical techniques. The point is that one can embed densely Aut $\left(\left\langle\mathbb{U}_{0},<\right\rangle\right)$, where $\left\langle\mathbb{U}_{0},<\right\rangle$ is as in the example in I) before, into Iso $(\mathbb{U})$ and then quote the extreme amenability of Aut $\left(\left\langle\mathbb{U}_{0},<\right\rangle\right)$, which is based on 4.3 and Nešetřil's result that finite ordered metric spaces have the Ramsey Property. Incidentally, it was this approach that led the authors of [30] to conjecture this Ramsey theorem, which was then proved by Nešetřil. It thus provides an example of an indirect use of these ideas in the discovery of new classes with the Ramsey Property.

III) Recently Bodirsky-Pinsker-Tsankov [9] used 4.3 to prove the following result, which they then applied to solve decision problems concerning definability in ultrahomogeneous structures. Another more direct proof was later found by Sokić.

Theorem 4.4 (Bodirsky-Pinsker-Tsankov [9]). Let $\boldsymbol{K}$ be an order Fraïssé structure, in a language $L$, whose age satisfies the RP. Let $n \geq 1$ and let $L_{n}=$ $L \cup\left\{c_{1}, \ldots, c_{n}\right\}$, where $c_{1}, \ldots, c_{n}$ are constant symbols. Let $a_{1}, \ldots, a_{n}$ be in $K$ and let $\boldsymbol{K}(\bar{a})=\left\langle\boldsymbol{K}, a_{1}, \ldots, a_{n}\right\rangle$ be the Fraïssé structure, in the language $L_{n}$, in which $c_{i}^{K(\bar{a})}=a_{i}$, for $1 \leq i \leq n$. Then the age of $\boldsymbol{K}(\bar{a})$ also satisfies the $\mathrm{RP}$.

See also the survey paper Bodirsky-Pinsker [8] for other consequences of 4.3. In particular the authors point out (see [8], Corollary 45) that given order Fraïssé classes $\mathcal{K}, \mathcal{K}^{\prime}$ in, e.g., a finite relational (i.e., one with no function symbols) language, for which their Fraïssé limits are bi-interpretable (in the sense of AhlbrandtZiegler [1]) $\mathcal{K}$ has the RP iff $\mathcal{K}^{\prime}$ has the RP. They discuss examples of how this fact can be used to deduce the RP for new classes and they also discuss its relevance to constraint satisfaction.

IV) Recall that a structure $\boldsymbol{K}$ is $\aleph_{0}$-categorical if all countable infinite models of the first-order theory of $\boldsymbol{K}$ are isomorphic. This will be the case, for example, if $\boldsymbol{K}$ is a Fraïssé structure in a finite relational language. We now have:

Theorem 4.5. Let $\boldsymbol{K}$ be an $\aleph_{0}$-categorical Fraïssé structure. Then $\boldsymbol{K}$ can be expanded to a Fraissé structure $\boldsymbol{K}^{\prime}=\left\langle\boldsymbol{K}, R_{i}\right\rangle_{i \in \mathbb{N}}$, with non-trivial automorphism group, by adding countably many relations, so that Age $\left(\boldsymbol{K}^{\prime}\right)$ has the Ramsey Property.

Bodirsky, Pinsker and Tsankov raised the very interesting question of whether this can be done using an expansion by only finitely many relations. 
D) We next discuss the effect of the duality theory to the problem of determining universal minimal flows.

Given a language $L$, consider the language $L^{*}=L \cup\{<\}$ obtained by adding a binary relation $<$ to $L$. Any structure $\boldsymbol{A}^{*}$ in $L^{*}$ is of the form $\boldsymbol{A}^{*}=\langle\boldsymbol{A},<\rangle$, where $\boldsymbol{A}$ is a structure for $L$ and $<\subseteq A^{2}$. It is an order structure if $<$ is a linear ordering of $A$. We also put $\boldsymbol{A}^{*} \mid L=\boldsymbol{A}$. Finally we say that a class of finite structures $\mathcal{K}^{*}$ in $L^{*}$, closed under isomorphism, is an order expansion of a class $\mathcal{K}$ of finite structures in $L$, if every $\boldsymbol{A}^{*} \in L^{*}$ is an order structure and $\mathcal{K}=\mathcal{K}^{*} \mid L=\left\{\boldsymbol{A}^{*} \mid L: \boldsymbol{A}^{*} \in \mathcal{K}^{*}\right\}$. If $\langle\boldsymbol{A},<\rangle \in \mathcal{K}^{*}$ we say that $<$ is a $\mathcal{K}^{*}$-admissible ordering on $\boldsymbol{A}$.

In the above notation, an order class $\mathcal{K}^{*}$ in $L^{*}$ is called reasonable if for every $\boldsymbol{A}, \boldsymbol{B} \in \mathcal{K}$, every embedding $\pi: \boldsymbol{A} \rightarrow \boldsymbol{B}$ and every $\mathcal{K}^{*}$-admissible ordering $<_{\boldsymbol{A}}$ on $\boldsymbol{A}$, there is a $\mathcal{K}^{*}$-admissible ordering $<_{\boldsymbol{B}}$ on $\boldsymbol{B}$ with $\pi$ also an embedding of $\boldsymbol{A}^{*}=\left\langle\boldsymbol{A},<_{\boldsymbol{A}}\right\rangle$ into $\boldsymbol{B}^{*}=\left\langle\boldsymbol{B},<_{\boldsymbol{B}}\right\rangle$ (i.e., $\pi$ also preserves $<_{\boldsymbol{A}},<_{\boldsymbol{B}}$ ).

Finally, again in the above notation, we say that the class $\mathcal{K}^{*}$ has the ordering property (OP) if for every $\boldsymbol{A} \in \mathcal{K}=\mathcal{K}^{*} \mid L$, there is $\boldsymbol{B} \in \mathcal{K}$ such that for every $\mathcal{K}^{*}$-admissible ordering $<_{\boldsymbol{A}}$ of $\boldsymbol{A}$ and $\mathcal{K}^{*}$-admissible ordering $<_{\boldsymbol{B}}$ on $\boldsymbol{B}$, if $\boldsymbol{A}^{*}=$ $\left\langle\boldsymbol{A},<_{\boldsymbol{A}}\right\rangle, \boldsymbol{B}^{*}=\left\langle\boldsymbol{B},<_{\boldsymbol{B}}\right\rangle$, then $\boldsymbol{A}^{*} \leq \boldsymbol{B}^{*}$.

This concept arose in work of Nešetrril-Rödl in the 1970's in structural Ramsey theory (see [42] and also [43], 5.2), where it plays an important role. For example, Nešetřil-Rödl showed that $\mathcal{K}^{*}=\{$ finite ordered graphs $\}$ has the ordering property and used this to calculate the Ramsey objects of $\mathcal{K}=\mathcal{K}^{*} \mid L=\{$ finite graphs $\}$, i.e., the finite graphs $\boldsymbol{A}$ that have the property that for any graph $\boldsymbol{B} \geq \boldsymbol{A}$ and any $r \geq 1$, there is a graph $\boldsymbol{C} \geq \boldsymbol{B}$ with $\boldsymbol{C} \rightarrow(\boldsymbol{B})_{r}^{\boldsymbol{A}}$. This concept is also crucial in calculating the so-called Ramsey degrees of structures in $\mathcal{K}$, for which we refer the reader to [12], [30] and references contained therein as well as the more recent [46].

If now $\mathcal{K}$ is a Fraïssé class in $L$ and $\mathcal{K}^{*}$ a reasonable, order expansion of $\mathcal{K}$ in $L^{*}$, let $\boldsymbol{K}=\operatorname{Flim}(\mathcal{K})$ and let $X_{\mathcal{K}^{*}}$ be the space of linear orderings $<$ on $K$ with the property that for any finite $\boldsymbol{A} \subseteq \boldsymbol{K},<\mid A$ is $\mathcal{K}^{*}$-admissible on $\boldsymbol{A}$. We call these the $\mathcal{K}^{*}$-admissible linear orderings on $\boldsymbol{K}$. They form a (nonempty) compact subspace of $2^{K^{2}}$ (with the product topology) on which $G=\operatorname{Aut}(\boldsymbol{K})$ acts continuously, i.e., $X_{\mathcal{K}^{*}}$ is a $G$-flow which is metrizable.

Here is then the next result in the duality theory that connects now the "calculation" of the universal minimal flow of non-archimedean groups represented as automorphism groups of Fraïssé structures $\boldsymbol{K}$ to the finite combinatorics of $\mathcal{K}=\operatorname{Age}(\boldsymbol{K})$.

Theorem 4.6 ([30]). Let $L$ be a language, $L^{*}=L \cup\{<\}, \mathcal{K}^{*}$ a reasonable Fraïssé order class in $L^{*}$. Let $\mathcal{K}=\mathcal{K}^{*} \mid L$. Then $\mathcal{K}$ is a Fraïsé class in $L$ and if $\boldsymbol{K}=$ $\operatorname{Flim}(\mathcal{K}), \boldsymbol{K}^{*}=\operatorname{Flim}\left(\mathcal{K}^{*}\right), \boldsymbol{K}=\boldsymbol{K}^{*} \mid L$. Moreover we have, letting $G=\operatorname{Aut}(\boldsymbol{K})$,

(i) $X_{\mathcal{K}^{*}}$ is a minimal $G$-flow iff $\mathcal{K}^{*}$ has the OP.

(ii) $X_{\mathcal{K}^{*}}$ is the universal minimal flow of $G$ iff $\mathcal{K}^{*}$ has the RP and the OP.

It is a heuristic principle in structural Ramsey theory that the RP for $\mathcal{K}^{*}$ as above is stronger than the OP and indeed in some cases one can give a proof of OP using RP. However there are well-known examples where RP is true but OP fails (see, e.g., [30], p. 163). The following result gives a precise formulation of 
this heuristic principle and shows a surprising uniqueness associated with OP. Its proof uses topological dynamics and is another application of duality.

Theorem 4.7 ([30]). Let $L$ be a language, $L^{*}=L \cup\{<\}, \mathcal{K}^{*}$ a reasonable Fraïssé order class in $L^{*}$. If $\mathcal{K}^{*}$ satisfies the $\mathrm{RP}$, then there is a reasonable Fraïssé order class $\mathcal{K}^{* *} \subseteq \mathcal{K}^{*}$ with $\mathcal{K}^{* *}\left|L=\mathcal{K}^{*}\right| L$, which satisfies both $\mathrm{RP}$ and $\mathrm{OP}$. Moreover $\mathcal{K}^{* *}$ is essentially unique (in a sense that is explained precisely in [30], 9.2).

It also follows from 4.6 and 4.7 that if $\mathcal{K}$ is a Fraïssé class in $L$ that admits a reasonable, Fraïssé order expansion $\mathcal{K}^{*}$ in $L^{*}$ with the $\mathrm{RP}$, then if $\boldsymbol{K}=\operatorname{Flim}(\mathcal{K})$ and $G=\operatorname{Aut}(\boldsymbol{K})$, the universal minimal flow of $G$ is metrizable.

We finally discuss some concrete applications of this result to the determination of the universal minimal flow of various non-archimedean groups. Many more such results can be found in [30], [46]. We keep the above notation.

(i) $\mathcal{K}=\{$ finite sets $\}, \mathcal{K}^{*}=\{$ finite linear orderings $\}, \boldsymbol{K}=\operatorname{Flim}(\mathcal{K})=\langle\mathbb{N}\rangle$, $X_{\mathcal{K}^{*}}=\mathrm{LO}(\mathbb{N})=$ the space of linear orderings on $\mathbb{N}, G=\operatorname{Aut}(\boldsymbol{K})=S_{\infty}$. The universal minimal flow of $S_{\infty}$ is $\mathrm{LO}(\mathbb{N})$ (Glasner-Weiss [16]).

(ii) $\mathcal{K}=\{$ finite graphs $\}, \mathcal{K}^{*}=\{$ finite ordered graphs $\}, \boldsymbol{K}=\operatorname{Flim}(\mathcal{K})=\boldsymbol{R}=$ the random graph, $X_{\mathcal{K}^{*}}=\mathrm{LO}(R), G=\operatorname{Aut}(\boldsymbol{R})$. The universal minimal flow of $\operatorname{Aut}(\boldsymbol{R})$ is $\mathrm{LO}(R)$.

(iii) $\mathcal{K}=\{$ finite-dimensional vector spaces over a finite field $F\}, \mathcal{K}^{*}=\{$ lex. ordered finite-dimensional vector spaces over $F\}, \boldsymbol{K}=\operatorname{Flim}(\mathcal{K})=\boldsymbol{V}_{\infty, F}=$ the countably infinite-dimensional vector space over $F, X_{\mathcal{K}^{*}}=\mathrm{LO}_{\text {lex }}\left(V_{\infty, F}\right)$ (the subspace of $\mathrm{LO}\left(V_{\infty, F}\right)$ consisting of all linear orderings whose restrictions to finitedimensional subspaces are lexicographic $), G=\mathrm{GL}\left(\boldsymbol{V}_{\infty, F}\right)$. The universal minimal flow of $\operatorname{GL}\left(\boldsymbol{V}_{\infty, F}\right)$ is $X_{\mathcal{K}^{*}}$.

(iv) $\mathcal{K}=\{$ finite metric spaces with rational distances $\}, \mathcal{K}^{*}=\{$ ordered metric spaces with rational distances $\}, \boldsymbol{K}=\operatorname{Flim}(\mathcal{K})=\boldsymbol{U}_{0}, X_{\mathcal{K}^{*}}=\mathrm{LO}\left(U_{0}\right), G=$ $\operatorname{Aut}(\boldsymbol{K})=\operatorname{Iso}\left(\boldsymbol{U}_{0}\right)$. The universal minimal flow of $\operatorname{Iso}\left(\boldsymbol{U}_{0}\right)$ is $\operatorname{LO}\left(U_{0}\right)$.

(v) $\mathcal{K}=\{$ finite posets $\}, \mathcal{K}^{*}=\{$ finite posets with linear extensions $\}$ (i.e., a structure $\langle P, \prec,<\rangle$ is in $\mathcal{K}^{*}$ if $\langle P, \prec\rangle$ is a poset and $<$ is a linear extension of $\prec$ ), $\boldsymbol{K}=\operatorname{Flim}(\mathcal{K})=$ the random poset $=\boldsymbol{P}, X_{\mathcal{K}^{*}}=$ the subspace of $\mathrm{LO}(P)$ consisting of all linear extensions of the partial order of $\boldsymbol{P}, G=\operatorname{Aut}(\boldsymbol{P})$. The universal minimal flow of $\operatorname{Aut}(\boldsymbol{P})$ is $X_{\mathcal{K}^{*}}$.

(vi) $\mathcal{K}=\{$ finite Boolean algebras $\}, \mathcal{K}^{*}=\{$ lex. ordered finite Boolean algebras $\}$, $\boldsymbol{K}=\boldsymbol{B}_{\infty}=$ the countable atomless Boolean algebra, $X_{\mathcal{K}^{*}}=$ the subspace of $\mathrm{LO}\left(B_{\infty}\right)$ consisting of all linear orderings whose restrictions to every finite Boolean algebra are lexicographical, $G=\operatorname{Aut}\left(\boldsymbol{B}_{\infty}\right) \cong H\left(2^{\mathbb{N}}\right)$, the homeomorphism group of the Cantor space. The universal minimal flow of $\operatorname{Aut}\left(\boldsymbol{B}_{\infty}\right)$ is $X_{\mathcal{K}^{*}}$. The universal minimal flow of $H\left(2^{\mathbb{N}}\right)$ was first calculated in Glasner-Weiss [17] as the so-called Uspenskij space. This is quite different but of course isomorphic to $X_{\mathcal{K}^{*}}$ above.

In recent work of Nguyen Van Thé [47] an extension of the preceding framework is developed which allows expansion of structures, called preceompact in [47], by more than linear orderings and this is important for further calculations of universal minimal flows, like that of the automorphism group of the dense local order. This is because in certain cases, like this one, we cannot find order expansions with 
the Ramsey Property. Another example of this is in Jasiński [28], who considers the class of boron trees. Finally Kechris-Sokic [32] deal with another situation that presents a similar difficulty. The Fraïssé class $\mathcal{D}$ of finite distributive lattices admits no Fraïssé order expansions but one can calculate the universal minimal flow of the automorphism group of its Fraïssé limit $\boldsymbol{D}$, which is called the random distributive lattice, by applying the preceding duality theory to an appropriate "cofinal" subclass of $\mathcal{D}$.

\section{Topological dynamics, II: Generic symmetries and auto- matic continuity phenomena}

A) Let $X$ be a topological space and $P \subseteq X$ a subset of $X$ viewed as a property of elements of $X$. As usual, we say that $P$ is generic if it is comeager in $X$. For example, nowhere differentiability is a generic property in $C([0,1])$. But what does it mean to say that an individual element $x_{0}$ of $X$ is generic?

Suppose now $X$ is a topological space equipped with a "natural" equivalence relation $E$. Then we view the relation $x E y$ as "identifying" in some sense $x$ and $y$. We then say that $x_{0} \in X$ is generic (relative to $E$ ) if the $E$-equivalence class of $x_{0}$ is comeager, i.e., the generic element of $X$ is $E$-equivalent, i.e., "identical" to $x_{0}$.

For example, if a group $G$ acts on $X$, then an element $x_{0} \in X$ is generic for this action if its orbit $G \cdot x_{0}$ is comeager. In the particular case when a topological group $G$ acts on itself by conjugation, we call $g_{0} \in G$ generic if its conjugacy class is comeager. Quite often groups we are interested in appear as groups of symmetries of mathematical structures, e.g., the homeomorphism group $H(X)$ of a compact metrizable space $X$, the unitary group $U(H)$ of separable Hilbert space $H$, the isometry group $\operatorname{Iso}(X, d)$ of a separable complete metric space $(X, d)$, the automorphism group $\operatorname{Aut}(X, \mu)$ of a standard measure space $(X, \mu)$ or the automorphism group $\operatorname{Aut}(\boldsymbol{K})$ of a countable structure $\boldsymbol{K}$. In these cases we talk about a generic symmetry of the structure.

When does a symmetry group $G$ have generic elements? We have here a vague dichotomy:

Symmetry groups of "continuous structures", like $U(H), \operatorname{Aut}(X, \mu), \ldots$ do not have generic elements.

Symmetry groups of "discrete structures", like Aut $(\boldsymbol{K}), \boldsymbol{K}$ a countable structure, often have generic elements.

We will next consider the problem of existence of generic automorphisms for Fraïssé structures and its implications. This kind of problem was first studied in model theory, see, e.g., Lascar [37], Truss [59], Hodges-Hodkinson-Lascar-Shelah [24]. It also arose in topological dynamics, e.g., in the work of Akin-HurleyKennedy [3], who studied generic properties of homeomorphisms of the Cantor space and asked whether it has a generic homeomorphism. By Stone duality this is equivalent to asking if there is a generic automorphism of the countable atomless Boolean algebra $\boldsymbol{B}_{\infty}$. 
We now discuss work of Kechris-Rosendal [31] on this problem.

Let $\mathcal{K}$ be a Fraïssé class with $\boldsymbol{K}=\operatorname{Flim}(\mathcal{K})$. Truss has associated with $\mathcal{K}$ a new class of finite objects $\mathcal{K}_{p}$ consisting of all pairs $\mathfrak{A}=(\boldsymbol{A}, \varphi: \boldsymbol{B} \rightarrow \boldsymbol{C})$, where $\boldsymbol{B}, \boldsymbol{C} \subseteq \boldsymbol{A}$ are in $\mathcal{K}$ and $\varphi$ is an isomorphism of $\boldsymbol{B}, \boldsymbol{C}$. There is a natural notion of embedding of objects in $\mathcal{K}_{p}$ and thus one can formulate the concept of joint embedding property (JEP) and amalgamation property (AP) for $\mathcal{K}_{p}$. Then Truss found a sufficient condition for the existence of a generic automorphism of $\boldsymbol{K}$ in terms of properties of $\mathcal{K}_{p}$.

Theorem 5.1 (Truss [59]). If $\mathcal{K}_{p}$ has a cofinal (under embedding) class $\mathcal{K}_{p}^{\prime} \subseteq \mathcal{K}_{p}$ that satisfies JEP and AP, then there is a generic automorphism of $\boldsymbol{K}$.

Truss [59] asked then for a necessary and sufficient condition. Using a dynamical point of view (applying Hjorth's concept of turbulence to the conjugacy action of $\operatorname{Aut}(\boldsymbol{K}))$ leads to an answer to this question.

We say that $\mathcal{K}_{p}$ has the weak amalgamation property (WAP) if it satisfies the following:

For any $\mathfrak{C} \in \mathcal{K}_{p}$, there is $\mathfrak{C}^{*} \in \mathcal{K}_{p}$ and an embedding $\pi: \mathfrak{C} \rightarrow \mathfrak{C}^{*}$ such that for any embeddings $f: \mathfrak{C}^{*} \rightarrow \mathfrak{A} g: \mathfrak{C}^{*} \rightarrow \mathfrak{B}$, with $\mathfrak{A}, \mathfrak{B} \in \mathcal{K}_{p}$, there is $\mathfrak{D} \in \mathcal{K}_{p}$ and embeddings $r: \mathfrak{A} \rightarrow \mathfrak{D}, s: \mathfrak{B} \rightarrow \mathfrak{D}$ with $r \circ f=s \circ g$.

We now have:

Theorem $5.2([31])$. Let $\mathcal{K}$ be a Fraïssé class and $\boldsymbol{K}=\operatorname{Flim}(\mathcal{K})$. Then $\boldsymbol{K}$ has a generic automorphism iff $\mathcal{K}_{p}$ has the JEP and WAP.

This result was also proved by Ivanov [27] in the case of $\aleph_{0}$-categorical $\boldsymbol{K}$ by different methods.

We next list some examples of structures that admit generic automorphisms.

(i) $\mathbb{N}$; (Kruske-Truss [34]) the random poset $\boldsymbol{P}$; (Truss [59]) $\langle\mathbb{Q},<\rangle$.

(ii) (Kechris-Rosendal [31]) the countable atomless Boolean algebra.

An equivalent formulation of (ii) is that there is a generic homeomorphism of the Cantor space. Akin-Glasner-Weiss [2] later found another, topological, proof of this result and gave a characterization of the properties of the generic homeomorphism.

We will discuss more examples of such structures later on but let us point out that there are also Polish groups that fail to be non-archimedean but still admit generic elements, e.g., the group of increasing homeomorphisms of [0,1] (see [31]).

B) We will next consider a multidimensional notion of genericity.

Definition 5.3. Let a topological group $G$ act on a topological space $X$. Then $G$ acts also co-ordinatewise on $X^{n}(n \geq 1)$ :

$$
g \cdot\left(x_{1}, \ldots, x_{n}\right)=\left(g \cdot x_{1}, \ldots, g \cdot x_{n}\right) .
$$

We say that $\left(x_{1}, \ldots, x_{n}\right) \in X^{n}$ is $n$-generic if it is generic for this action. We finally say that the action of $G$ on $X$ has ample generics if for each $n \geq 1$, there is an n-generic element of $X^{n}$. Applying this to the conjugacy action of a topological group $G$ on itself, we say that $G$ has ample generics if for each $n \geq 1$, there is $\left(g_{1}, \ldots, g_{n}\right) \in G^{n}$ such that $\left\{\left(g g_{1} g^{-1}, \ldots, g g_{n} g^{-1}\right): g \in G\right\}$ is comeager in $G^{n}$. 
For automorphism groups of countable structures, this concept also first came up in model theory (see [24]). We note here that it is impossible to have "infinite generics" for any non-trivial Polish group, i.e., generics for $G^{\mathbb{N}}$ (see [31], p. 331).

It is now known that there are many examples of countable structures that admit ample generic automorphisms (i.e., their automorphism groups admit ample generics). These include:

(i) $\mathbb{N}$,

(ii) the random graph $\boldsymbol{R}$ (Hrushovski [25]),

(iii) many automorphism groups of $\omega$-stable, $\aleph_{0}$-categorical structures (HodgesHodkinson-Lascar-Shelah [24]),

(iv) $\mathbb{U}_{0}$ (Solecki [57]),

(v) the infinitely-splitting rooted tree ([31]).

Also the group of measure-preserving homeomorphisms of the Cantor space admits ample generics ([31]).

However until very recently it remained open whether the countable atomless Boolean algebra admits ample generic automorphisms or equivalently whether the homeomorphism group of the Cantor space admits ample generics. This was finally resolved positively by Kwiatkowska [35], who used the powerful tools of the theory of dual or projective Fraïssé limits developed in Irwin-Solecki [26].

There are also structures that admit generics but not ample generics. An interesting example is $\langle\mathbb{Q},<\rangle$ which admits generics but not even 2-generics (Hodkinson, see [60] and [56]).

An important open problem here is whether there exist Polish groups that fail to be non-archimedean but have ample generics.

It is also unknown whether there is a non-trivial Polish locally compact group which has a generic element. If has been pointed out by K.H. Hofmann that if such a group exists, there will be also one which is non-archimedean.

C) It turns out that Polish groups with ample generics have many remarkable properties and we will discuss this next.

\section{1) The small index property}

A Polish group has the small index property (SIP) if every subgroup of index $<2^{\aleph_{0}}$ is open.

Thus for non-archimedean groups SIP implies that the topology of the group is determined by its algebra. Hodges-Hodkinson-Lascar-Shelah [24] used (special types of) ample generics to prove SIP for automorphism groups of certain structures. It turns out that this is a general phenomenon.

Theorem 5.4 ([31]). If a Polish group $G$ has ample generics, then $G$ has the SIP.

\section{2) Automatic continuity}

Automatic continuity results have been proved in several instances for various types of algebras but until recently there were only few examples of this phenomenon for groups and then under rather severe restrictions on the types of groups. Building on the earlier work of [24] it was shown in [31] that this phenomenon holds in a very general framework in the presence of ample generics. 
Theorem 5.5 ([31]). Let $G$ be a Polish group that admits ample generics. Then any algebraic homomorphism $\pi: G \rightarrow H$, where $H$ is a separable topological group, is continuous.

In particular such groups have a unique Polish (group) topology. For $S_{\infty}$ one actually has a stronger result.

Theorem 5.6 ([31]). The group $S_{\infty}$ has a unique non-trivial separable group topology.

One can now view the proof of 5.5 as factored through a property referred to as the Steinhaus property, introduced in Rosendal-Solecki [55].

Definition 5.7. A Polish group has the Steinhaus property (SP) if there is $n \geq 1$ such that for any $A \subseteq G$ with $1 \in A=A^{-1}$, which is countably (left) syndetic (i.e., countably many left translates of $A$ cover $G$ ), 1 is contained in the interior of $A^{n}$.

It can be seen that 5.5 holds for any Polish group $G$ with the SP and then it is shown that any Polish group with ample generics has the SP.

Using ample generics (and some variations) it is now known that many other Polish groups satisfy the SP and thus the phenomenon of automatic continuity is quite pervasive. Here are some examples:

(i) (Rosendal-Solecki $[55]) H\left(2^{\mathbb{N}}\right),\left(H\left(2^{\mathbb{N}}\right)\right)^{\mathbb{N}}, \operatorname{Aut}(\langle\mathbb{Q},<\rangle), H(\mathbb{R})$.

(ii) (Rosendal [53]) $H(M)$, where $M$ is a compact 2-manifold.

(iii) (Kittrell-Tsankov [33]) The full group $[E]$ of an ergodic, measure preserving countable Borel equivalence relation on a standard measure space $(X, \mu)$.

(iv) (Ben Yaacov-Berenstein-Melleray [6]) The automorphism group $\operatorname{Aut}(X, \mu)$ of a standard measure space.

(v) (Tsankov [61]) The unitary group $U(H)$ of the infinite-dimensional separable Hilbert space $H$.

The last two results also use the theory of "topometric ample generics" due to Berenstein, Ben Yaacov and Melleray [6]. An extensive survey of automatic continuity for groups can be found in Rosendal [54].

Remark 5.8. An interesting corollary of the automatic continuity for the group $\operatorname{Aut}(\langle\mathbb{Q},<\rangle)$ is that, when viewed as a discrete group, it satisfies the fixed point on metric compacta property. Other groups with this property include $\operatorname{Aut}(X, \mu)$ and $U(H)$ (see [6], [61]).

\section{3) The Bergman property}

Bergman [7] introduced the following "finite generation" property, now known as the Bergman Property, and proved that it holds for $S_{\infty}$.

Definition 5.9. A group $G$ has the Bergman Property (BP) if for any increasing sequence $E_{n}, n \geq 1$, of subsets of $G$ with $G=\bigcup_{n} E_{n}$, there is $n, k$ with $G=\left(E_{n}\right)^{k}$.

This is equivalent to each one of the following:

(i) (a) $G$ has uncountable cofinality, i.e., cannot be written as a union of an increasing sequence of proper subgroups, and (b) if $1 \in S \subseteq G$ is a symmetric generating set, then for some $n, G=S^{n}$.

(ii) Every action of $G$ by isometries on a metric space has a bounded orbit. 
A closed subgroup $G$ of $S_{\infty}$ is oligomorphic if the action of $G$ on $\mathbb{N}^{n}, n \geq 1$, has only finitely many orbits. Equivalently, these are exactly the automorphism groups of $\aleph_{0}$-categorical countable structures (see, e.g., Cameron [11]).

Theorem 5.10 ([31]). Let $G$ be an oligomorphic, closed subgroup of $S_{\infty}$ with ample generics. Then $G$ has the Bergman property.

There are now known many other examples of groups with the Bergman Property. For example:

(i) (de Cornulier - see [10]) $H\left(S^{n}\right)$.

(ii) (Ricard-Rosendal [52]) $U(H)$.

(iii) (Miller [40]) Aut $(X, \mu)$.

4) Other properties of groups with ample generics, like uncountable cofinality, Serre's property (FA), etc., can be also found in [31].

\section{Ergodic theory, I: Unique ergodicity phenomena}

(A) We will next discuss very recent work in progress of Angel-Kechris-Lyons on the ergodic theory of non-archimedean Polish groups.

Let $G$ be a Polish group and $X$ a $G$-flow. We will be looking at invariant Borel probability measures on $X$. In general such measures might not exist.

Definition 6.1. A topological group $G$ is called amenable if every $G$-flow admits an invariant Borel probability measure.

In particular every extremely amenable group is amenable. But the infinite symmetric group $S_{\infty}$ is amenable but not extremely amenable and the automorphism group of the countable atomless Boolean algebra, i.e., $H\left(2^{\mathbb{N}}\right)$, is not amenable.

A particularly important class of automorphism groups that are amenable is the following:

Definition 6.2. Let $\mathcal{K}$ be a Fraïssé class of finite structures. We say that $\mathcal{K}$ is a Hrushovski class if for any $\boldsymbol{A} \in \mathcal{K}$ there is $\boldsymbol{B} \in \mathcal{K}$ with $\boldsymbol{A} \subseteq \boldsymbol{B}$ such that every partial automorphism of $\boldsymbol{A}$ (i.e., an isomorphism between substructures of $\boldsymbol{A})$ extends to a (full) automorphism of $\boldsymbol{B}$.

This can be viewed as a "finitization" of the ultrahomogeneity of $\operatorname{Flim}(\mathcal{K})$. Some basic examples of Hrushovski classes are the pure sets, graphs (Hrushovski [25]), rational valued metric spaces (Solecki [57]), finite-dimensional vector spaces over a finite field, etc.

Definition 6.3. Let $\mathcal{K}$ be a Fraïssé class of finite structures with $\operatorname{Flim}(\mathcal{K})=\boldsymbol{K}$. If $\mathcal{K}$ is a Hrushovski class, we say that $\boldsymbol{K}$ is a Hrushovski structure.

This turns out to be a property of automorphism groups. 
Proposition 6.4 ([31]). Let $\mathcal{K}$ be a Fraïssé class of finite structures with $\boldsymbol{K}=$ $\operatorname{Flim}(\mathcal{K})$. Then the following are equivalent:

(i) $\boldsymbol{K}$ is a Hrushovski structure.

(ii) $\operatorname{Aut}(\boldsymbol{K})$ is compactly approximable, i.e., there is an increasing sequence $\left(K_{n}\right)$ of compact subgroups of $G$ with $\bigcup_{n} K_{n}$ dense in $G$.

From this it easily follows that the automorphism group of any Hrushovski structure is amenable.

Definition 6.5. If $G$ is an amenable group and $X$ a $G$-flow, we say that this flow is uniquely ergodic if there is a unique invariant Borel probability measure on $X$.

In this case the unique measure must be ergodic (i.e., $G$-invariant Borel sets are null or co-null for this measure).

Definition 6.6. If $G$ is a Polish amenable group, we say that $G$ is uniquely ergodic if every minimal $G$-flow has a unique invariant probability measure.

Trivially every extremely amenable Polish group is uniquely ergodic and so is every compact Polish group. It follows from results in [64] that every countable infinite group admits minimal actions with many invariant measures and Benjamin Weiss believes that this can be extended to non-compact, locally compact groups although this has not been checked in detail yet. It turns out that many amenable automorphism groups of Fraïssé structures, for which we have been able to calculate a metrizable universal minimal flow, are uniquely ergodic, so this may be a general phenomenon.

In fact we do not know any counterexample to the following very (perhaps ridiculously) strong conjecture.

Conjecture 6.7 (Unique Ergodicity Conjecture). Let $G$ be a non-archimedean Polish group with metrizable universal minimal flow. If $G$ is amenable, then it is uniquely ergodic.

Even if false in this generality, it is conceivable that some restricted version of it might still be true. Here are some groups for which this conjecture has been verified.

Theorem 6.8. The following groups are uniquely ergodic:

(i) (Glasner-Weiss [16]) $S_{\infty}$.

(ii) (Angel-Kechris-Lyons [4])

(1) $\operatorname{Aut}(\boldsymbol{R}), \operatorname{Aut}\left(\boldsymbol{H}_{n}\right)$, where $\boldsymbol{R}$ is the random graph and $\boldsymbol{H}_{n}$ the random $n$-regular hypergraph.

(2) $\operatorname{Aut}(\boldsymbol{E})$, where $\boldsymbol{E}$ is the equivalence relation on $\mathbb{N}$ with infinitely many classes, all infinite.

(3) $\operatorname{Aut}\left(\boldsymbol{T}_{\infty}\right)$, the automorphism group of the rooted $\aleph_{0}$-regular tree. This is isomorphic to the isometry group of the Baire space $\mathcal{N}$ with the usual metric. 
(4) For countable $S \subseteq(0, \infty)$, the isometry group of the Urysohn ultrametric space $\boldsymbol{U}_{S}$ with distances in $S$.

(5) The general linear group of the countably infinite-dimensional vector space $\boldsymbol{V}_{\infty, F}$ over a finite field $F$.

(B) Interestingly it turns out that unique ergodicity fits well in the duality theory discussed in $\S 4$. For Hrushovski classes it is in fact equivalent to a quantitative version of the Ordering Property.

Let $\mathcal{K}$ be a Hrushovski class that admits a reasonable Fraïssé order expansion $\mathcal{K}^{*}$ (e.g., $\mathcal{K}=\{$ graphs $\}, \mathcal{K}^{*}=\{$ ordered graphs $\}$ ). Then the Ordering Property for $\mathcal{K}^{*}$ can be expressed as follows:

(*) Given a structure $\boldsymbol{A}$ in $\mathcal{K}$, there is a structure $\boldsymbol{B}$ in $\mathcal{K}$ with $\boldsymbol{A} \subseteq \boldsymbol{B}$ such that for every pair of $\mathcal{K}^{*}$-admissible orderings $<_{\boldsymbol{A}}$ on $\boldsymbol{A}$ and $<_{\boldsymbol{B}}$ on $\boldsymbol{B}$ there is an automorphism $\pi$ of $\boldsymbol{B}$ with $\pi\left(<_{\boldsymbol{A}}\right) \subseteq<_{\boldsymbol{B}}$.

If now $\boldsymbol{K}=\mathrm{Flim}(\mathcal{K})$ and $\mathcal{K}^{*}$ is reasonable and has both $\mathrm{RP}$ and $\mathrm{OP}$, we have seen in $\S 4$ that $X_{\mathcal{K}^{*}}$ is the universal minimal flow of $G=\operatorname{Aut}(\mathcal{K})$. The unique ergodicity of $G$ is equivalent to the unique ergodicity of $X_{\mathcal{K}^{*}}$. Using this one can show the following:

Theorem 6.9 (Angel-Kechris-Lyons [4]). Let $\mathcal{K}$ be a Hrushovski class with $\boldsymbol{K}=$ $\operatorname{Flim}(\mathcal{K})$ and let $\mathcal{K}^{*}$ be a reasonable Fraïssé order expansion of $\mathcal{K}$ which satisfies $\mathrm{RP}$ and $\mathrm{OP}$. Then the following are equivalent

(i) $G=\operatorname{Aut}(\boldsymbol{K})$ is uniquely ergodic.

(ii) There is a map $\boldsymbol{A}^{*}=\langle\boldsymbol{A},<\rangle \in \mathcal{K}^{*} \mapsto \rho\left(\boldsymbol{A}^{*}\right) \in(0,1]$ which is isomorphism invariant and such that for any $\boldsymbol{A} \in \mathcal{K}, \epsilon>0$, there is $\boldsymbol{B} \in \mathcal{K}, \boldsymbol{B} \supseteq \boldsymbol{A}$, so that for every $\mathcal{K}^{*}$-admissible ordering $<_{\boldsymbol{A}}$ on $\boldsymbol{A}$ and $\mathcal{K}^{*}$-admissible ordering $<_{\boldsymbol{B}}$ on $\boldsymbol{B}$, we have

$$
\left|\frac{\left|\left\{\pi \in \operatorname{Aut}(\boldsymbol{B}): \pi\left(<_{\boldsymbol{A}}\right) \subseteq<_{\boldsymbol{B}}\right\}\right|}{|\operatorname{Aut}(\boldsymbol{B})|}-\rho(\boldsymbol{A},<)\right|<\epsilon .
$$

For example, in the case $\mathcal{K}=\{$ finite graphs $\}$, unique ergodicity of the automorphism group of the random graph is equivalent to the statement that for any finite graph $\boldsymbol{A}$ with $n$ vertices and any $\epsilon>0$, there is a finite graph $\boldsymbol{B} \supseteq \boldsymbol{A}$ such that for any ordering $<_{\boldsymbol{A}}$ on $\boldsymbol{A}$ and $<_{\boldsymbol{B}}$ on $\boldsymbol{B}$ the ratio of the automorphisms of $\boldsymbol{B}$ that preserve $<_{\boldsymbol{A}},<_{\boldsymbol{B}}$ over the number of all automorphisms of $\boldsymbol{B}$ is, up to $\epsilon$, equal to $1 / n$ !. In this case one can also show that any finite graph $\boldsymbol{A}$ with $n$ vertices and any $\epsilon>0$, there is a finite graph $\boldsymbol{B}$ such that for any orderings $<_{\boldsymbol{A}}$, $<_{\boldsymbol{B}}$ on $\boldsymbol{A}, \boldsymbol{B}$, resp., the ratio of the number of embeddings of $\boldsymbol{A}$ into $\boldsymbol{B}$ that preserve the orderings $<_{\boldsymbol{A}},<_{\boldsymbol{B}}$ over the number of all embeddings of $\boldsymbol{A}$ into $\boldsymbol{B}$ is, up to $\epsilon$, equal to $1 / n$ !.

A random, consistent ordering on the class of finite graphs is a map that assigns to each finite graph $\boldsymbol{A}$ a probability ordering $\mu_{\boldsymbol{A}}$ on the set $\operatorname{LO}(A)$ of linear orderings on $A$, which is isomorphism invariant, and satisfies that for any finite graphs $\boldsymbol{A} \subseteq \boldsymbol{B}$, if $\pi: \mathrm{LO}(B) \rightarrow \mathrm{LO}(A)$ is the projection map $\pi(<)=<\mid A$, then $\pi$ sends $\mu_{\boldsymbol{B}}$ to $\mu_{\boldsymbol{A}}$.

An example of such a random ordering is the uniform ordering, where for any $\boldsymbol{A}$ with $n$ vertices $\mu_{\boldsymbol{A}}(<)=1 / n$ !. Now it turns out that the unique ergodicity 
of the automorphism group of the random graph is equivalent to the statement that the uniform ordering is the unique random, consistent ordering on the class of finite graphs, so this last statement is true.

(C) There is an even stronger form of 6.7 that specifies the support of the unique invariant measure, namely that, under the assumptions of 6.7 , the unique invariant measure is supported by a single orbit, which is moreover dense $G_{\delta}$.

This was shown to be true for $S_{\infty}$ in Glasner-Weiss [16] and more generally by Angel-Kechris-Lyons [4] for uniquely ergodic $\operatorname{Aut}(\boldsymbol{K})$, where $\boldsymbol{K}=\operatorname{Flim}(\mathcal{K})$ for a Fraïssé class $\mathcal{K}$ which admits a Fraïssé order expansion $\mathcal{K}^{*}$ with the RP and OP such that for $\boldsymbol{A} \in \mathcal{K}$ every linear ordering on $A$ is $\mathcal{K}^{*}$-admissible. This includes, for example, the automorphism groups of the random graph and $n$-regular hypergraph.

(D) One very interesting area of research concerning the ergodic theory of $S_{\infty}$, that we will not be able to discuss here, has to do with the study and classification of invariant measures of the so-called logic action of $S_{\infty}$, i.e., the canonical action of $S_{\infty}$ on the space of countable structures (with universe $\mathbb{N}$ ) of some fixed language (and some of its invariant subspaces). This is related to exchangeability theory, a branch of probability and ergodic theory which is an outgrowth of the classical de Finetti theorem. Notable results in this theory include contributions by Aldous, Austin, Diaconis, Hoover, Janson, Kallenberg, Petrov and Vershik for graphs and hypergraphs, where important connections exist with the theory of graph limits developed by Borgs, Chayes, Lovász, Sós, Szegedy, Vesztergombi and others.

An important recent development in this area is the work of Ackerman-FreerPatel, who, extending work of Petrov-Vershik [51], provided a necessary and sufficient condition for the existence of invariant measures on the isomorphism class of the Fraïssé limit of a class of finite structures $\mathcal{K}$, in terms of a strong amalgamation property. It appears that this is a very fruitful area of interactions between model theory, ergodic theory, probability theory and combinatorics.

\section{Ergodic theory, II: Spatial realizations}

Let $(X, \mu)$ be a standard measure space, i.e., $X$ is a standard Borel space and $\mu$ a Borel probability measure on $X$. Let $\mathrm{MEAS}_{\mu}$ be the measure algebra of $(X, \mu)$, i.e., the Boolean algebra of Borel sets of $X$ modulo $\mu$-null sets with the usual topology given by the metric $d_{\mu}(A, B)=\mu(A \Delta B)$. Let also $\operatorname{Aut}(X, \mu)$ be the group of automorphisms of $(X, \mu)$ with the weak topology (see, e.g., Kechris [29]) in which it is a Polish group.

Given a Polish group $G$ a measure preserving Boolean action of $G$ on $(X, \mu)$ is a continuous action of $G$ on $\mathrm{MALG}_{\mu}$ or equivalently a continuous homomorphism of $G$ into $\operatorname{Aut}(X, \mu)$. A spatial model of such an action is a Borel action of $G$ on $X$ such that for each Borel set $A \subseteq X$ and $g \in G$ we have $[g \cdot A]_{\mu}=g \cdot[A]_{\mu}$, where $[A]_{\mu}$ is the element of $\mathrm{MALG}_{\mu}$ represented by $A$.

Let us say that a Polish group $G$ has the Mackey Property (MP) if every measure preserving Boolean action of $G$ admits a spatial realization. The name is justified by the following classical result. 
Theorem 7.1 (Mackey [39]). Every Polish locally compact group has the MP.

On the other hand there are Polish groups failing the MP, the group $\operatorname{Aut}(X, \mu)$ being one of them (Becker, Glasner-Tsirelson-Weiss [15]). See Glasner-Weiss [18] and Glasner-Tsirelson-Weiss [15] for a penetrating analysis of this problem.

It turns out that all non-archimedean groups have the MP.

Theorem 7.2 (Glasner-Weiss [18]). Let $G$ be a Polish non-archimedean group. Then $G$ has the Mackey Property.

More recently this result was extended to a much larger class of Polish groups that include those in 7.1 and 7.2 (e.g., it includes closed subgroups of countable products of locally compact Polish groups).

Theorem 7.3 (Kwiatkowska-Solecki [36]). Let $G$ be a Polish group of isometries of a locally compact separable metric space. Then $G$ has the Mackey Property.

We refer to [36] and the references contained therein for more on this spatial realization problem.

\section{Unitary representations}

Let $G$ be a Polish group. A (continuous) unitary representation of $G$ is a continuous action of $G$ on a (complex) Hilbert space $H$ by unitary transformations. It is irreducible if it has no non-trivial closed (linear) subspaces.

A goal of representation theory is to describe (up to isomorphism) the irreducible representations and understand how other representations are built out of the irreducible ones.

A classical example of such an analysis is the Peter-Weyl Theorem for compact groups.

Theorem 8.1 (Peter-Weyl). Let $G$ be a compact Polish group. Then

(i) There are only countably many irreducible unitary representations and every unitary representation of $G$ is a direct sum of irreducible representations.

(ii) The irreducible unitary representations are all finite dimensional and the left-regular representation of $G$ is the direct sum of all the irreducible representations, each appearing with multiplicity equal to its dimension.

Recently Tsankov [62] proved a remarkable analog of Peter-Weyl for oligomorphic closed subgroups of $S_{\infty}$, which are far from compact. This is a significant extension of earlier results that were proved by different methods for $S_{\infty}$ itself and (a variant of) $\mathrm{GL}\left(\boldsymbol{V}_{\infty, F}\right)$, see Lieberman [38] and Olshansky [48].

Recall here that a closed subgroup of $S_{\infty}$ is oligomorphic if its action on any $\mathbb{N}^{n}, n \geq 1$, has only finitely many orbits. Equivalently these are the automorphism groups of $\aleph_{0}$-categorical (Fraïssé) structures. For example the automorphism group of any relational Fraïssé structure in a finite language has this property. 
Theorem 8.2 (Tsankov [62]). Let $G$ be an oligomorphic closed subgroup of $S_{\infty}$. There are only countably many irreducible unitary representations of $G$ and every unitary representation of $G$ is a direct sum of irreducible representation.

We note however that in general these irreducible representations are not finitedimensional.

Moreover, Tsankov [62] provides in many cases an explicit description of the irreducible representations. For example, for the automorphism group of the random graph one obtains all the irreducible representations by "lifting" through the process of induction the irreducible representations of the automorphism groups of finite graphs. Also for $\operatorname{Aut}(\langle\mathbb{Q},<\rangle)$ the irreducible representations are exactly the actions of this group on $\ell^{2}\left([\mathbb{Q}]^{n}\right)$, where $[\mathbb{Q}]^{n}=\{X \subseteq \mathbb{Q}:|X|=n\}, n \geq 1$.

Finally Tsankov [62] uses his analysis to show that many oligomorphic groups have Kazhdan's Property (T).

Definition 8.3. A topological group $G$ has property (T) if there is compact $Q \subseteq G$ and $\epsilon>0$ such that every unitary representation of $G$ that has a unit $(Q, \epsilon)$ invariant vector has actually a unit invariant vector. (A unit vector $v$ is $(Q, \epsilon)$ invariant if $\|g \cdot v-v\|<\epsilon, \forall g \in Q$.)

Tsankov shows that such groups include $S_{\infty}, \operatorname{Aut}(\boldsymbol{R}), \operatorname{Aut}\left(\boldsymbol{B}_{\infty}\right), \operatorname{Aut}(\langle\mathbb{Q}$, $<\rangle), \operatorname{GL}\left(\boldsymbol{V}_{\infty, F}\right)$.

\section{References}

[1] G. Ahlbrandt and M. Ziegler, Quasi-finitely axiomatizable totally categorical theories. Ann. Pure Appl. Logic 30(1) (1986), 63-82..

[2] E. Akin, E. Glasner and B. Weiss, Generically there is but one self homeomorphism of the Cantor set. Trans. Amer. Math. Soc. 360(7) (2008), 3613-3630.

[3] E. Akin, M. Hurley and J. A. Kennedy, Dynamics of topologically generic homeomorphisms. Mem. Amer. Math. Soc. 783 (2003).

[4] O. Angel, A.S. Kechris and R.D. Lyons, paper in preparation.

[5] H. Becker and A.S. Kechris, The descriptive set theory of Polish group actions. London Math. Society Lecture Note Series 232, Cambridge Univ. Press, 1996.

[6] I. Ben Yaacov, A. Berenstein and J. Melleray, Polish topometric groups. arXiv:1007.3367v3 (2010).

[7] G. M. Bergman, Generating infinite symmetric groups. Bull. London Math. Soc. 38 (2006), 429-440.

[8] M. Bodirsky and M. Pinsker, Reducts of Ramsey structures. Contemp. Math. 558 (2011), 489-519.

[9] M. Bodirsky, M. Pinsker and T. Tsankov, Decidability of definability. arXiv:1012.2381v3 (2011).

[10] D. Calegari and M. Freedman, Distortion in transformation groups. With an appendix by Yves de Cornulier. Geom. Topol. 10 (2006), 267-293. 
[11] P.J. Cameron, Oligomorphic permutation groups. London Math. Society Lecture Note Series 152, Cambridge Univ. Press, 1990.

[12] W.L. Fouché, The Ramsey degrees of finite relational structures. J. Comb. Theory Ser. A 85(2) (1999), 135-147.

[13] T. Giordano and V. Pestov, Some extremely amenable groups related to operator algebras and ergodic theory. J. Inst. Math. Jussieu 6 (2007), 279-315.

[14] E. Glasner, On minimal actions of Polish groups. Top. Appl. 85 (1998), 119-125.

[15] E. Glasner, B. Tsirelson and B. Weiss, The automorphism group of the Gaussian measure cannot act pointwise. Israel J. Math. 148 (2005), 305-329.

[16] E. Glasner and B. Weiss, Minimal actions of the group $S(\mathbb{Z})$ of permutations of the integers. Geom. Funct. Anal. 12 (2002), 964-988.

[17] E. Glasner and B. Weiss, The universal minimal system for the group of homeomorphisms of the Cantor set. Fund. Math. 176 (2003), 277-289.

[18] E. Glasner and B. Weiss, Spatial and non-spatial actions of Polish groups. Ergod. Th. Dynam. Sys. 25 (2005), 1521-1538.

[19] R.L. Graham, K. Leeb and B.L. Rothschild, Ramsey's theorem for a class of categories. Adv. in Math. 8 (1972), 417-433.

[20] R.L. Graham and B.L. Rothschild, Ramsey's theorem for $n$-parameter sets. Trans. Amer. Math. Soc. 159 (1971), 257-292.

[21] E. Granirer and A.T. Lau, Invariant means on locally compact groups. Ill. J. Math. 15 (1971), 249-257.

[22] M. Gromov and V.D. Milman, A topological application of the isoperimetric inequality. Amer. J. Math. 105 (1983), 843-854.

[23] W. Herer and J.P.R. Christensen, On the existence of pathological submeasures and the construction of exotic topological groups. Math. Ann. 213 (1975), 203-210.

[24] W. Hodges, I. Hodkinson, D. Lascar and S. Shelah, The small index property for $\omega$-stable $\omega$-categorical structures and for the random graph. J. London Math. Soc. 48(2) (1993), 204-218.

[25] E. Hrushovski, Extending partial isomorphisms of graphs. Combinatorica 12 (1992), 411-416.

[26] T. Irwin and S. Solecki, Projective Fraïssé limits and the pseudo-arc. Trans. Amer. Math. Soc. 358(7) (2006), 3077-3096.

[27] A. A. Ivanov, Generic expansions of $\omega$-categorical structures and semantics of generalized quantifiers. J. Symbolic Logic 64 (1999), 775-789.

[28] J. Jasiński, Ramsey degrees of boron tree structures. Preprint (2010).

[29] A.S. Kechris, Global aspects of ergodic group actions. Math. Surveys and Monographs 160, Amer. Math. Soc., 2010.

[30] A.S. Kechris, V. Pestov and S. Todorcevic, Fraïssé limits, Ramsey theory, and topological dynamics of automorphism groups. Geom. Funct. Anal. 15 (2005), 106-189.

[31] A.S. Kechris and C. Rosendal, Turbulence, amalgamation, and generic automorphisms of homogeneous structures. Proc. London Math. Soc. 93(3) (2007), 302-350.

[32] A.S. Kechris and M. Sokić, Dynamical properties of the random poset and random distributive lattice. Preprint (2011). 
[33] J. Kittrell and T. Tsankov, Topological properties of full groups. Ergod. Th. Dynam. Syst. 30(2) (2010), 525-545.

[34] D. Kuske and J. K. Truss, Generic automorphisms of the universal partial order. Proc. Amer. Math. Soc. 129 (2001), 1939-1948.

[35] A. Kwiatkowska, The group of homeomorphisms of the Cantor set has ample generics. arXiv:1104.3340 (2011).

[36] A. Kwiatkowska and S. Solecki, Spatial models of Boolean actions and groups of isometries. Ergod. Th. Dynam. Syst. 31(2) (2011), 405-421.

[37] D. Lascar, Les beaux automorphismes. Arch. Math. Logic 31 (1991), 55-68.

[38] A. Lieberman, The structure of certain unitary representations of infinite symmetric groups. Trans. Amer. Math. Soc. 164 (1972), 189-198.

[39] G. W. Mackey, Point realisations of transformation groups. Illinois J. Math. 6 (1962), 327-335.

[40] B. Miller, Full groups, classification, and equivalence relations. Ph.D.Thesis, U.C. Berkeley (2004).

[41] T. Mitchell, Fixed points and multiplicative left invariant means. Trans. Amer. Math. Soc. 122 (1966), 195-202

[42] J. Nešetřil, On ordered graphs and graph orderings. Discrete Appl. Math. 51 (1994), 113-116.

[43] J. Nešetřil, Ramsey theory. Handbook of Combinatorics (ed. by R.L. Graham et al.) Elsevier, 1995, 1331-1403.

[44] J. Nešetřil, Metric spaces are Ramsey. European J. Combin. 28(1) (2007), 457-468.

[45] J. Nešetřil and V. Rödl, Partitions of finite relational and set systems. J. Combin. Theory 22(3) (1977), 289-312.

[46] L. Nguyen Van Thé, Structural Ramsey theory of metric spaces and topological dynamics of isometry groups. Memoirs Amer. Math. Soc. 968 (2010).

[47] L. Nguyen Van Thé, More on the Kechris-Pestov-Todorcevic correspondence: precompact expansions. arXiv:1201.1270v1 (2011).

[48] G. I. Olshansky, Unitary representations of the infinite symmetric group: a semigroup approach. Representations of Lie groups and Lie algebras (Budapest, 1971) (1985), 18-197.

[49] V. Pestov, On free actions, minimal flows and a problem of Ellis. Trans. Amer. Math. Soc. 350(10) (1998), 4149-4165.

[50] V. Pestov, Ramsey-Milman phenomenon, Urysohn metric spaces, and extremely amenable groups. Israel J. Math. 127 (2002), 317-357.

[51] F. Petrov and A. Vershik, Uncountable graphs and invariant measures on the set of universal countable graphs. Random Structures Algorithms 37(3) (2010), 389-406.

[52] E. Ricard and C. Rosendal, On the algebraic structure of the unitary group. Collect. Math. 58(2) (2007), 181-192,

[53] C. Rosendal, Automatic continuity in homeomorphism groups of compact 2manifolds. Israel J. Math. 166 (2008), 349-367.

[54] C. Rosendal, Automatic continuity of group homomorphisms. Bull. Symbolic Logic 15(2) (2009), 184-214. 
[55] C. Rosendal and S. Solecki, Automatic continuity of homomorphisms and fixed points on metric compacta. Israel J. Math. 162 (2007), 349-371.

[56] K. Slutsky, Non-genericity phenomenon in some ordered Fraïssé classes. arXiv:1010.0462 (2010).

[57] S. Solecki, Extending partial isometries. Israel J. Math. 150 (2005), 315-331.

[58] S. Thomas, Groups acting on infinite dimensional projective spaces. J. London Math. Society 34(2) (1986), 265-273.

[59] J. K. Truss, Generic automorphisms of homogeneous structures. Proc. London Math. Soc. 65(3) (1992), 121-141.

[60] J.K. Truss, On notions of genericity and mutual genericity. J. Symb. Logic 72(3) (2007), 755-766.

[61] T. Tsankov, Automatic continuity for the unitary groups. arXiv:1109.1200 (2011).

[62] T. Tsankov, Unitary representations of oligomorphic groups. arXiv:1101.2194v1 (2011).

[63] W. Veech, Topological dynamics. Bull. Amer. Math. Soc. 83(5) (1977), 775-830.

[64] B. Weiss, Minimal models for free actions. preprint (2011).

Alexander S. Kechris, Department of Mathematics, California Institute of Technology, Pasadena, CA 91125, USA

E-mail: kechris@caltech.edu 